\title{
A study of the positive benefits of bandwidth feedback in the learning process of motor skills
}

\section{Fan Zhang}

Department of Police Skills and Tactics, Nanjing Forest Police College, Nanjing 210023, China

Sports Science postdoctoral programme, Nanjing Normal University, Nanjing 210023, China

zhangfan@nfpc.edu.cn

Keywords: bandwidth feedback, motor skill learning, learning process, positive benefits.

\begin{abstract}
: the current research of bandwidth feedback has positive benefits in motor learning, such as providing feedback for motor performance outside the bandwidth, giving full play to the function of feedback in information, making the learners modify the motor according to the error information provided by the quantitative feedback, and advance the motor performance, and to the bandwidth table. The present feedback provides qualitative information, in addition to avoiding the unstable movements caused by overcorrection, and enhancing the performance of correct movements. In order to further clarify the progress of the research, this study reviews the research on the performance of motor skill learning with bandwidth feedback in order to provide a scientific reference for the follow-up study.
\end{abstract}

\section{Introduction}

Feedback is one of the main topics in the research field of motor skill learning. After an motor is executed, people can understand the process and results of the motor through information provided by various sensory perceptions or additional information provided by the outside world. Therefore, feedback can be divided into intrinsic feedback, also called intrinsic feedback, and extrinsic feedback, also called augmented feedback; extrinsic feedback is divided into knowledge of performance (KP) and knowledge of results (KR) based on what it provides. Magill pointed out in view of the information value of intrinsic feedback and extrinsic feedback that when intrinsic feedback cannot provide sufficient information, the demand of motor executors for extrinsic feedback in terms of information increase accordingly [1]. Hence, the discussion of feedback must involve the characteristics of motor itself in terms of intrinsic feedback and the ability of learners to perceive intrinsic feedback information, which means that the provision of augmented feedback is not absolutely necessary. However, the acquisition of feedback has the information function of providing movement correction and control after movement execution, stimulating learning motivation, and enhancing the connection function of correct movement stimulus and response, thus affecting movement displaying and learning.

In techniques that are influenced by the $G$ hypothesis (guidance hypothesis) to reduce the frequency of motor-learning feedback, the way in which motor performance is provided as a basis for feedback is called bandwidth feedback. Feedback provides a way as a basis for augmented feedback through a predetermined range of motor performance. Traditional bandwidth feedback provides quantitative results (knowledge of results, KR) in which the absolute error of the motor performance falls outside the set bandwidth, and feedback provides guidance for motor correction [2]. If the motor performance falls within the bandwidth it does not provide feedback, or provides qualitative feedback, because the learners have been told before the experiment that the meaning of the expression falls to the target range, which means that the motor is performed correctly and is with positive feedback information as well as the motivation function of feedback. Bandwidth settings also have the role of goal setting. 


\section{Theoretical Basis}

The theoretical basis of bandwidth feedback for feedback frequency and the timing of feedback provision is the blocking hypothesis [3]. If the frequency of augmented feedback provision is too high, or the timing of the provision is inappropriate, it will be detrimental to the effect of motor learning, because high frequency feedback provision encourage learners to rely on extrinsic feedback information, and hinder the processing of intrinsic feedback information. If the time interval of the feedback provision is too short, there will not be sufficient time for learners to deal with the feedback information, so learners can not improve the ability of errors detection. Therefore, the way of bandwidth setting can reduce the frequency of KR, and avoid the inappropriate timing and practice of feedback, which interferes with the learners' learning of intrinsic feedback information. Another theoretical basis for bandwidth feedback is themaladaptive short-term correction hypothesis, saying that augmented feedback has a role of motivation, encouraging learners to correct motors based on feedback provided by the information. However, for the motors without KR, the learner tends to repeat the motor, and reduce the degree of motor correction, and then produce stable motor performance. It is conducive to the stability of motor characterization acquisition, so high-frequency feedback, according to the $G$ hypothesis, makes learners ignore the intrinsic feedback information processing, and also causes too much motor correction as well as interferes with learning. Providing qualitative feedback information or not providing feedback after the motor with minor error can avoid the learner's frequent motor correction for minor errors, but it causes the reduce in motor stability and the variation between the motors increase; the negative effect of the motor learning on the stability is produced. In short, in addition to reducing the frequency of feedback to prevent learners from relying on feedback, the more important significance of the bandwidth feedback is that when the motor is approaching the target, providing the qualitative feedback is helpful for stabilizing the performance of the motor, avoiding unnecessary corrective motor, and providing positive information, which has the effect of motivation. While the quantified feedback information is provided for the learner with greater errors in practice, the motor-guided correction of reference information, is provided only when learners are in poor performance and fall behind the scope of the test. The implementing principle of bandwidth feedback takes learners' motor performance during the acquisition period as the reference mode of feedback provision.

\section{Research Progress}

According to the theory of the G hypothesis, at present, the research confirms that the bandwidth feedback is helpful to the motor learning. The previous studies have found that the larger the bandwidth setting range is, the lower the frequency of the quantitative feedback is, the better performance the test has. Sherwood found in the arm's moving angle that the $10 \% \mathrm{BW}$ group was better than 5\% BW and 0\% BW group in maintaining the stability. Lai and Shea found that the motor learning of $15 \% \mathrm{BW}$ was superior to that of $0 \% \mathrm{BW}$ in the sequence key work [4]. Badets and Blandin examined the observation and learning effects of bandwidth feedback in key timing work [5], and found that the bandwidth feedback in observational learning had the effect of motor learning. Smith, Taylor and Withers examined the motor of golf's playing and found that the stability of $10 \% \mathrm{BW}$ was better than that of $5 \% \mathrm{BW}$ group and $0 \% \mathrm{BW}$ group [6]. CocaUgrinowitsch and Ugrinowitsch in the study of darts, found that the motor learning effect of bandwidth feedback was better than the control group [7], and the same motor learning effect was also confirmed in the study of golf's playing [8]. Sadowski, Mastale and Niznikowski examined the relatively complex gymnastic skills and provided bandwidth feedback through errors in the performance of key movements to inform (knowledge of performance, KP), and also discovered the learning effects of bandwidth feedback [9].

The above researches supported the effect of bandwidth feedback on motor learning, so specific bandwidth feedback studies were incidental to the study of motor learning variables. Cauraugh, 
Chen and Radlo tested arm movement and confirmed the motor learning effect of $10 \%$ BW group [10]. They also tested the effect of feedback content on the change of motor correction and the results showed that the guidance effect of the quantitative feedback information on the motor performance change was better than the qualitative feedback, but in the case of large motor error, no matter when qualitative or quantitative feedback was provided, the motor change degree of the subsequent exercise was larger than that of the relatively small error. Goodwin and Meeuwsen tested golf's playing and found that the learning effect of $10 \%$ BW group was better than that of $0 \%$ BW group [11]. The study also aimed at the distribution of feedback frequency. By the grouping design of shrinking-BW and expanding-BW, it was found that the performance of the extended bandwidth group was better than that of the reduced bandwidth group in the absence of $\mathrm{KR}$, and the study showed the advantages of bandwidth feedback in the feedback frequency control of the motor learning. Butler, Reeve and Fischman tested the time-sequence rocker's shifting and found the effect of $14 \%$ BW group's motor learning was better than that of the control group. It was also found that the learning effect of traditional bandwidth feedback group could not be produced without informing learners not to provide feedback about correct motor. Lee and Carnahan tested the case where they did not inform the learner not providing feedback for the correct motor and found that the learning effect of bandwidth feedback was worse than the traditional bandwidth feedback, and the factors that the bandwidth feedback was beneficial to motor learning were not only the frequency of reducing feedback, but also the function of providing the qualitative feedback motivation in the bandwidth. Patterson and Azizieh found through the feedback provided by learners after relatively good performanec, the group told with good feedback had better effect of motor learning than that of not being told [12]. For the provision of qualitative or quantitative feedback in both intrinsic and extrinsic bandwidth, Wright, Smith-Munyon and Sidaway believed that qualitative feedback did not show a correction direction, so it had no significant effect on the guidance of motor correction, but it led to stable motor performance, while quantitative feedback information provided explicit indication information of error direction and error value, which was helpful to be the reference of motor correction [13]. For the accuracy of the feedback information provided, Magill and Wood pointed out that in the acquisition period, simply providing the correct qualitative feedback information can not play an effective role in guiding motor, and it had insignificant effect than correct quantitative feedback information in terms of the motor learning effect. Thus, it would completely reduce the accuracy of feedback information lacked the proper accurate feedback information, and could not produce effective motor learning effect. Therefore, in terms of the implementation of the traditional bandwidth feedback, the group with poor motor performance should be provided with the correct quantitative feedback information and providing qualitative feedback for the group with better performance was beneficial to the repetition of the relative correct motor.

Based on the existing bandwidth feedback research, it can be found that the bandwidth feedback has a positive effect in motor learning. If the feedback is provided on the basis of the motor performance outside the bandwidth, it can play the role of feedback in information, so that learners can carry out motor correction based on quantitative feedback provided by the error message of motor performance, and thus enhance the performance of motor. However, providing qualitative information based on the feedback of the performance inside bandwidth, not only avoids the learners' unstable motor due to excessive motor correction, but also enhances the performance of the correct motor. However, previous studies have found that the bandwidth value of the best learning effect is somewhat different and may be influenced by the characteristics of the learner's own conditions and movements. In addition, the implementation of bandwidth feedback, which provides corrected quantitative feedback for poorly performed motors or provides qualitative feedback for better performed motors, facilitates the repetition of relatively correct motors. The combination of qualitative and quantitative feedback has a positive effect on the formation of motor skills. 


\section{Conclusion}

According to the above bandwidth feedback research, the bandwidth feedback has positive effect on the motor learning, including reducing the feedback frequency to reduce learners' reliance on the extrinsic feedback and improving intrinsic feedback information processing. It not only exercise the error detection ability, but also indirectly causes the movement study. Bandwidth feedback provides feedback on performance outside of bandwidth, the function of feedback in information can enable learners to provide accurate motor performance-error-related information according to quantitative feedback, perform motor correction to improve motor performance, and provide qualitative information for feedback in the bandwidth. It can not only avoid the learners' too much motor correction, but also be advantageous to the movement stability. Moreover, qualitative feedback information or not providing feedback, also enhances relatively correct movement performance, and has the effect of feedback enhancement and motivation. However, previous studies have found that the bandwidth value of the best learning effect is somewhat different and may be influenced by the characteristics of the learner's own conditions and movements, so bandwidth settings should be adjusted to take into account the learner's age, skill level, and intrinsic feedback information generated by the job execution.

\section{Acknowledgements}

This work was supported in part by the Project of the Fundamental Research Funds for the Central Universities under Grant LGZD201805, in part by Jiangsu University Philosophy and Social Sciences Research Fund Project under Grant 2017SJB0590, in part by the 13th Five-Year plan project of Jiangsu Education Science under Grant C-c/2018/01/11, in part by Nanjing Forest police College Teaching Reform Project under Grant ZD18104, in part by the Project of China Postdoctoral Science Foundation under Grant 2017M611849, in part by Jiangsu Qing Lan Project under Grant 2017, and in part by Pre-research project of Nanjing Forest police College under Grant LGY201603.

\section{References}

[1] Magill, R. A. (2011). Motor learning and control: Concepts and applications. New York: McGraw-Hill.

[2] Sherwood, D. E. (1988). Effect of bandwidth knowledge of results on movement consistency. Perceptual and Motor Skills, 66, 535-542.

[3] Schmidt, R. A., \& Lee, T. D. (2011). Motor control and learning: A behavioral emphasis (5th ed.). Champaign, IL: Human Kinetics.

[4] Lai, Q., \& Shea, C. H. (1999). The role of reduced frequency of knowledge of results during constant practice. Research Quarterly for Exercise and sport, 70, 33-40.

[5] Badets, A., \& Blandin, Y. (2005). Observational learning: Effects of bandwidth knowledge of results. Journal of Motor Behavior, 37, 211-216.

[6] Smith, P. J. K., Taylor, S. J., \& Withers, K. (1997). Applying bandwidth feedback scheduling to a golf shot. Research Quarterly for Exercise and Sport, 68, 215-221.

[7] Coca-Ugrinowitsch, A. A., \& Ugrinowitsch, H. (2014). Bandwidth knowledge of results on the learning of the saloon dart throwing task. Perceptual and Motor Skills, 118, 462-474.

[8] Bagherli, J., Mohsenzadeh, M., Sabzi, A. H., \& Fotrousi, F. (2013). Bandwidth feedback: Does it effective when provided near or far from target on learning of putting skill? Middle-East Journal of Scientific Research, 13, 1-4.

[9] Sadowski, J., Mastaler, A., Niznikowski, T. (2013). Benefits of bandwidth feedback in learning 
a complex gymnastic skill. Journal of Human Kinetics, 37, 183-193.

[10] Cauraugh, J. H., Chen, D., \& Radlo, S. J. (1993). Effects of traditional and reversed bandwidth knowledge of results on motor learning. Research Quarterly for Exercise and Sport, 64, 413-417.

[11] Goodwin, J. E., Meeuwsen, H. J. (1995). Using bandwidth knowledge of results to alter relative frequencies during motor skill acquisition. Research Quarterly for Exercise and Sport, 66, 99-104.

[12] Patterson, J. T., \& Azizieh, J. (2012). Knowing the good from bad: Does being awareof KR content matter? Human Movement Science, 31, 1449-1458.

[13] Wright, D. L., Smith-Munyon, V. L., \& Sidaway, B. (1997). How close is too close for precise knowledge of results? Research Quarterly for Exercise and Sport, 68, 172-176. 